\title{
RankNAS: Efficient Neural Architecture Search by Pairwise Ranking
}

\author{
Chi Hu${ }^{1}$, Chenglong Wang ${ }^{1}$, Xiangnan $\mathrm{Ma}^{1}$, Xia Meng ${ }^{1}$, \\ Yinqiao $\mathbf{L i}^{1}$, Tong Xiao ${ }^{1,2}{ }^{*}$ Jingbo $\mathbf{Z h u}^{1,2}$ Changliang $\mathbf{L i}^{3}$ \\ ${ }^{1}$ NLP Lab, School of Computer Science and Engineering \\ Northeastern University, Shenyang, China \\ ${ }^{2}$ NiuTrans Research, Shenyang, China \\ ${ }^{3}$ Kingsoft AI Lab, Beijing, China \\ huchinlp@gmail.com, clwang1119@gmail.com, \\ \{xiaotong, zhujingbo\}@mail.neu.edu.cn
}

\begin{abstract}
This paper addresses the efficiency challenge of Neural Architecture Search (NAS) by formulating the task as a ranking problem. Previous methods require numerous training examples to estimate the accurate performance of architectures, although the actual goal is to find the distinction between "good" and "bad" candidates. Here we do not resort to performance predictors. Instead, we propose a performance ranking method (RankNAS) via pairwise ranking. It enables efficient architecture search using much fewer training examples. Moreover, we develop an architecture selection method to prune the search space and concentrate on more promising candidates. Extensive experiments on machine translation and language modeling tasks show that RankNAS can design high-performance architectures while being orders of magnitude faster than state-ofthe-art NAS systems.
\end{abstract}

\section{Introduction}

Neural Architecture Search (NAS) has advanced state-of-the-art on various tasks, such as image classification (Zoph et al., 2018; Pham et al., 2018; Real et al., 2019; Tan et al., 2019), machine translation (Fan et al., 2020; So et al., 2019), and language modeling (Pham et al., 2018; Liu et al., 2019; Jiang et al., 2019; Li et al., 2020). Despite the remarkable results, conventional NAS methods are computationally expensive, requiring training millions of architectures during search. For instance, obtaining a state-of-the-art machine translation model with an evolutionary algorithm requires more than 250 GPU years (So et al., 2019).

Several techniques have been proposed to improve the search efficiency, such as sharing parameters among all architectures (Pham et al., 2018; Cai et al., 2018; Zhong et al., 2018), predicting the performance instead of full training (Liu et al., 2018;

${ }^{*}$ Corresponding author.

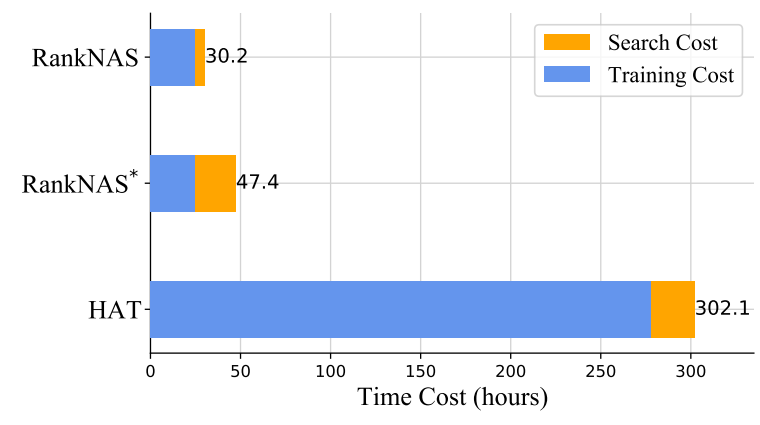

Figure 1: The time cost of different NAS methods on the WMT machine translation task. RankNAS* denotes the results without search space pruning. Our method significantly accelerates NAS through pairwise ranking and search space pruning.

Baker et al., 2018; Wen et al., 2020; Wei et al., 2020), and searching over a continuous space (Liu et al., 2019; Jiang et al., 2019; Li et al., 2020). Unfortunately, these approaches still suffer from the high cost of predicting the performance of each candidate architecture. An inherent reason for this is that obtaining accurate performance requires training numerous neural networks to convergence, as described in Sec. 2.2. However, it is unnecessary to predict the model performance as in previous NAS methods. Rather, all we need is to distinguish architectures of different quality in NAS, say, ranking these architectures.

In this paper, we approach the problem by formulating NAS as a ranking task. Here we propose RankNAS, a ranking model for comparing different architectures. One of the key challenges is that directly ranking all architectures in a large search space is still computationally infeasible. Therefore, we adopt the pairwise method (Burges et al., 2005; Wauthier et al., 2013), where the ranking problem is reduced to a binary classification problem over architecture pairs. To speed up RankNAS further, we develop an architecture selection method that chooses the most promising architectures for evalu- 
ation according to the importance of features, e.g., the topology of architectures.

We test RankNAS on well-established machine translation and language modeling benchmarks. Experiments show that RankNAS is orders of magnitude faster than standard NAS systems and can find better architectures. Notably, RankNAS is generic to different tasks and evaluation metrics. It achieves competitive results on hardware-aware NAS tasks and is $10 \times$ faster than the HAT baseline (Wang et al., 2020). It also discovers new architectures that outperform vanilla Transformer by +1.8 BLEU points on the IWSLT'14 De-En data and +1.5 BLEU points on the WMT'14 En-De data, surpassing the Evolved Transformer (So et al., 2019) with $150,000 \times$ less search cost.

\section{Preliminaries}

NAS generally consists of two steps: 1) sample architectures from the pre-defined search space, and 2) estimate the performance of these samples. This work focuses on the performance estimation step, which is the efficiency bottleneck of NAS.

\subsection{Search Space}

The search space contains all possible architectures for the search. In this work, we take the Transformer architecture for description, but the discussed problem and solutions are general and can be applied to other models. Following HAT (Wang et al., 2020), we represent a Transformer architecture as a set of features and search for the optimal model configuration.

An overview of the search space is shown in Figure 2. It is extended from the HAT's space and inspired by manually designed Transformer variants, including Relative Position Representations (Shaw et al., 2018) and Deep Transformer (Wang et al., 2019). The search space can also be represented as a supernet where each sub-network is a unique architecture. The search space contains around $10^{23}$ possible architectures, as detailed in Appendix A.1. It is computationally prohibited to explore such a large space with an exhaustive method.

\subsection{Performance Estimation}

Let $\mathcal{A}$ denotes the search space, and each architecture in it is represented by a feature vector $\alpha$. Formally, the goal of NAS is to find the optimal architecture $\alpha^{*}$ with the best performance. The per-

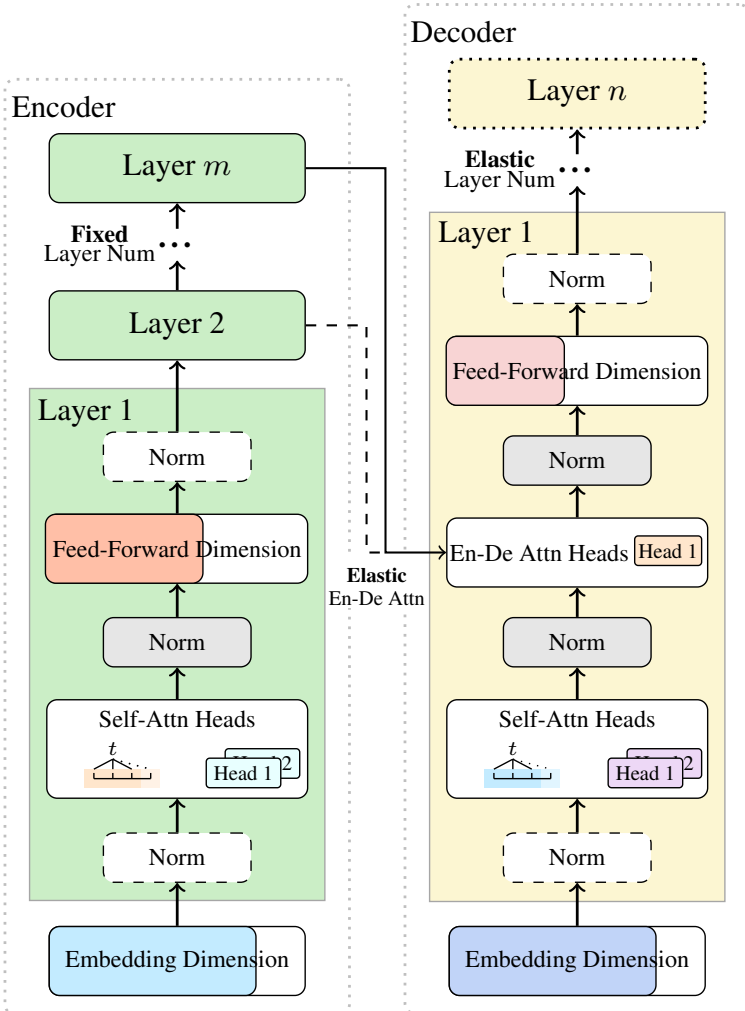

Figure 2: The architecture search space. We search for the optimal model size, e.g., the number of layers, and network topology, e.g., connections between different layers. The encoder part is ignored in the language modeling task. Appendix A.1 gives more details about the design choices for different tasks.

formance can be measured by some metrics, such as accuracy or latency. The performance estimation process consists of two steps: 1) estimate the performance of all architectures, and 2) choose the architecture with the optimal performance.

Without loss of generality, we define $\mathcal{S}(\cdot)$ as the performance evaluated by some metrics. The task here is to find the most promising architecture with maximum $\mathcal{S}(\cdot)$. Standard NAS methods solve this problem by learning to estimate the performance of each architecture. The objective is given by:

$$
\begin{aligned}
\alpha^{*} & =\underset{\alpha}{\operatorname{argmax}} \mathcal{S}_{\text {val }}\left(w^{*}, \alpha\right) \\
\text { s.t. } & w^{*}=\underset{w}{\operatorname{argmax}} \mathcal{S}_{\text {train }}(w, \alpha)
\end{aligned}
$$

where $w$ is the weights associated with the architecture. $\mathcal{S}_{\text {val }}$ and $\mathcal{S}_{\text {train }}$ are the evaluation results on the validation set and training set, respectively.

Optimizing Eq. 1 is time-consuming as obtaining the optimal weights for each architecture requires training them to converge. Although we can share the weights among all architectures to 
amortize the cost, performance evaluation is still nontrivial and requires numerous training steps.

\section{NAS as Ranking}

As mentioned in Sec. 2.2, the goal of NAS is to find promising architectures that achieve high performance on unseen data. NAS requires distinguishing whether the architectures are "good" or "bad" rather than predicting accurate performance. Therefore, it is natural to treat NAS as a ranking problem, in which the explicit goal is to rank different architectures correctly.

\subsection{Pairwise Ranking}

Problem Formulation. Given an architecture $\alpha$, we define a score $s$ on it by a function $r(\cdot)$ :

$$
s=r(\alpha, p)
$$

where $p$ is the parameter of the scoring function. We implement the scoring function with a gradient boosting decision tree, as detailed in Sec. 4.1.

We want to optimize $p$ such that $s$ assigns high scores to good architectures and low scores to bad architectures. This induces a ranking of the candidate architectures in the search space. It is infeasible to sort all candidate architectures in a large search space directly. A solution is to reduce the listwise ranking problem to the pairwise ranking problem. Fortunately, the properties of the NAS task allow us to achieve the goal. As described in Dudziak et al. (2020), the relation between any pair of performance is antisymmetric, transitive and connex. This makes it possible to rank all architectures via pairwise comparisons, substantially reducing the training complexity.

Training Set Construction. In pairwise ranking, the learning task is framed as a binary classification of architecture pairs into two categories: correctly ordered and incorrectly ordered. Given an architecture pair $\left(\alpha_{i}, \alpha_{j}\right)$ and the order of performance $\bar{P}_{i j}$, we can construct training examples $\left(\alpha_{i}, \alpha_{j}, \bar{P}_{i j}\right)$ for the classification by comparing the two values. Note that $\bar{P}_{i j}$ is a $0-1$ variable. For example, if $\alpha_{i}$ is better than $\alpha_{j}$, we would add $\left(\alpha_{i}, \alpha_{j}, 1\right)$ and $\left(\alpha_{j}, \alpha_{i}, 0\right)$ to the training set.

Optimization. Consider a pair of architectures $\left(\alpha_{i}, \alpha_{j}\right)$, scored by $s_{i}$ and $s_{j}$, respectively. The probability of $\alpha_{i}$ being better than $\alpha_{j}$ is given by the difference through an activation function $g$ :

$$
P_{i j}=g\left(s_{i}-s_{j}\right)
$$

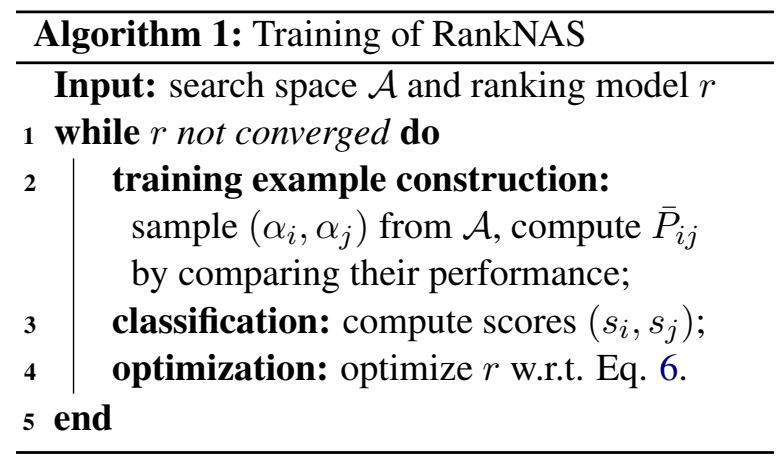

We assume that $P_{i j} \geq 0.5$ means $\alpha_{i}$ is better than $\alpha_{j}$ while $P_{i j}<0.5$ means $\alpha_{j}$ is better than $\alpha_{i}$. Here we use a logistic function to achieve this goal:

$$
P_{i j}=\frac{1}{1+e^{-\left(s_{i}-s_{j}\right)}}
$$

Similarly, $P_{j i}$ can be induced by:

$$
P_{j i}=\frac{1}{1+e^{-\left(s_{j}-s_{i}\right)}}=1-P_{i j}
$$

Denote the gold score of $\alpha_{i}$ being better than $\alpha_{j}$ as $\bar{P}_{i j}$. We use the cross-entropy loss function for the classification. The loss for a pair of inputs is:

$$
\begin{aligned}
L_{i j}= & -\left(\bar{P}_{i j} \log P_{i j}+\bar{P}_{j i} \log P_{j i}\right) \\
= & \left(1-\bar{P}_{i j}\right) \cdot\left(s_{i}-s_{j}\right)+ \\
& \log \left(1+e^{-\left(s_{i}-s_{j}\right)}\right)
\end{aligned}
$$

Compared with Eq. 1, Eq. 6 just requires $\bar{P}_{i j}$. In particular, we use the intermediate performance measured on the validation set during training. It is much easier than assessing the accurate performance of candidate architectures. In this sense, the ranking model is "easier" to learn and may not need many training samples as in performance prediction. RankNAS also enables efficient optimization through gradient methods. Algorithm 1 describes the complete training process of the ranking model.

\subsection{Applying Pairwise Ranking}

Although the training time of the ranking model is heavily reduced, it is still challenging to apply it to the ranking of all architectures in the search space $\mathcal{A}$. The challenge is that exploring all architectures is computationally expensive, even when the task is a binary classification.

Correlations between Features and Performance. We start by analyzing the effect of architectural features on estimated performance. Figure 4 illustrates the impact of the FFN dimension 


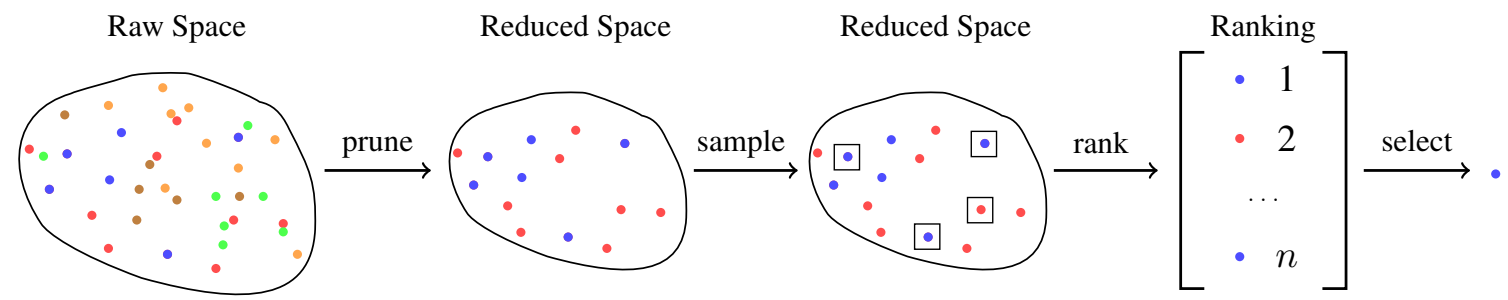

Figure 3: The proposed search process consists of three steps: 1) prune the search space according to the importance of architectural features, 2) sample $n$ architectures from the reduced search space by specific strategies, and 3) rank them with the trained ranking model and choose the best one. Here different color means different features.
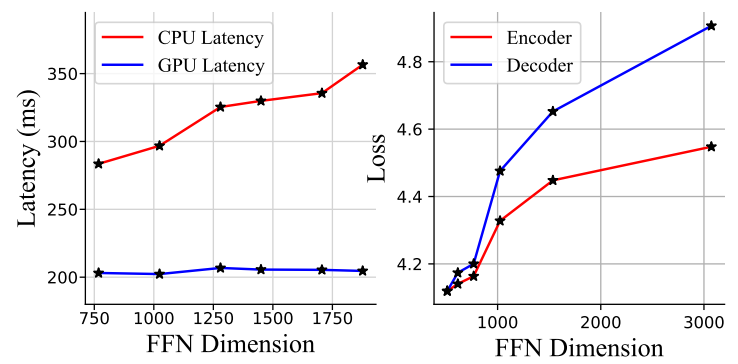

Figure 4: The impact of FFN dimension on latency and validation loss. All results are obtained on the WMT'14 En-De task with the same settings described in Sec. 4.2.

on latency and the validation loss on the machine translation task. We observe that: (a) different architectural features have very different correlations with the same evaluation metric, and (b) the same features also have different influences on different metrics. For example, the latency monotonically increases when scaling the FFN dimension on CPUs, while it is almost unchanged on GPUs. Hence, it is natural to improve search efficiency by eliminating unimportant features.

Feature Importance. Inspired by previous feature selection methods (Breiman, 2001; Fisher et al., 2019), we measure the importance of an architectural feature (e.g., the number of layers) by calculating the increase in the model error after permuting the feature.

We assume that each architecture $\alpha$ is represented by a feature vector $\boldsymbol{f} \in \mathcal{R}^{M \times N}$, where $M$ is the number of different features, and $N$ is the dimension of feature vectors. Also, we assume a set $C$ that contains $n$ architectures sampled from the search space. We first estimate the original model error $L_{\text {total }}$ on $C$ using the accumulation of the prediction errors. For any feature $\boldsymbol{f}_{i} \in \boldsymbol{f}$, we randomize it for each architecture in $C$. Then the randomized architectural features are passed to the ranking model and yield an error $L_{i}$. The importance of the $i$-th feature $f_{i}$ is defined by:

$$
I\left(\boldsymbol{f}_{i}\right)=\frac{L_{i}}{L_{\text {total }}}
$$

where a higher value implies $\boldsymbol{f}_{i}$ is more important.

Search Space Pruning. It is easy to select valuable architectural features with the above measure. Given all features $f \in \mathcal{R}^{M \times N}$, we discard those with a score less than a threshold $\theta$ and obtain the selected features $\boldsymbol{f}^{\prime} \in \mathcal{R}^{M^{\prime} \times N}$, where $M^{\prime}<M$. Then we can prune the search space according to the selected features. For instance, if the feature Embedding Dimension is not selected, we will keep it fixed during the search. Finally, we only search over the architectures in the reduced search space.

An overview of the search process is presented in Figure 3. As described in Sec. 3.1, the training of the proposed ranking model is much cheaper than previous methods, which need to optimize the parameters for all architectures. Pruning search space further reduces the number of architectures to be evaluated. Also, the sampling procedure can be implemented with any existing NAS search strategy, e.g., Random Search (RS) or Evolution Algorithm (EA).

\section{Experiments}

\subsection{Experimental Setups}

We evaluate our methods on language modeling and machine translation tasks. In the experiments, we search for hardware-aware architectures and high-accuracy architectures.

Training Setups. For machine translation, we experiment on the IWSLT'14 De-En and WMT'14 En-De tasks using the identical settings as Wang et al. (2020). For language modeling, we experiment on the WikiText-103 dataset (Merity et al., 


\begin{tabular}{|c|c|c|c|c|c|c|c|}
\hline Hardware & Task & Method & $\begin{array}{l}\text { Latency } \\
(\mathrm{ms})\end{array}$ & \#Params & $\begin{array}{l}\text { FLOPs } \\
\text { (G) }\end{array}$ & BLEU & $\begin{array}{c}\text { Search Cost } \\
\text { (hours) }\end{array}$ \\
\hline \multirow{6}{*}{$\begin{array}{l}\text { Intel Xeon } \\
\text { Silver } 4114 \\
\text { CPU }\end{array}$} & \multirow{3}{*}{ WMT } & Transformer & 1031.4 & $213.0 \mathrm{M}$ & 12.7 & 28.4 & - \\
\hline & & HAT & 396.8 & $67.9 \mathrm{M}$ & 4.2 & 28.5 & 335.1 \\
\hline & & RankNAS & 384.2 & $68.1 \mathrm{M}$ & 4.0 & 28.6 & 31.8 \\
\hline & \multirow{3}{*}{ IWSLT } & Transformer & 353.5 & $34.9 \mathrm{M}$ & 1.6 & 34.4 & . \\
\hline & & HAT & 190.5 & 27.9M & 1.4 & 34.5 & 31.7 \\
\hline & & RankNAS & 197.4 & $29.6 \mathrm{M}$ & 1.5 & 34.6 & 7.2 \\
\hline \multirow{6}{*}{$\begin{array}{l}\text { NVIDIA } \\
\text { GTX 1080Ti } \\
\text { GPU }\end{array}$} & \multirow{3}{*}{ WMT } & Transformer & 249.6 & $213.0 \mathrm{M}$ & 12.7 & 28.4 & - \\
\hline & & HAT & 214.8 & $66.2 \mathrm{M}$ & 4.1 & 28.5 & 302.1 \\
\hline & & RankNAS & 201.7 & $62.1 \mathrm{M}$ & 3.9 & 28.4 & 30.2 \\
\hline & \multirow{3}{*}{ IWSLT } & Transformer & 200.9 & $34.9 \mathrm{M}$ & 1.6 & 34.4 & - \\
\hline & & HAT & 159.4 & 33.9M & 1.6 & 34.7 & 24.5 \\
\hline & & RankNAS & 148.2 & $35.4 \mathrm{M}$ & 1.4 & 34.7 & 5.8 \\
\hline
\end{tabular}

Table 1: Comparisons of latency, model size, FLOPs, BLEU, and the overall search cost on machine translation tasks for the standard Transformer, HAT, and discovered architectures by our method. We mark the best results in bold for all metrics. Search costs are measured on a single RTX 2080Ti GPU.

2017) with the same settings as Baevski and Auli (2019). We set the maximum number of tokens per sample to 1,843 to fit the memory constraints and apply gradient accumulation to keep the same batch size as Baevski and Auli (2019) 's work. All models are trained with mixed precision on 8 NVIDIA RTX 2080 Ti GPUs except for IWSLT ones, which only take one GPU for training.

Ranking Model Setups. We implement the ranking model (binary classifier) described in Sec.3.1 with LightGBM (Ke et al., 2017) and set the learning rate to 0.1 . To prevent overfitting, we set the maximum number of leaves to 30 and the tree's maximum depth to 6 . We also use the default regularization terms and apply the early stopping strategy to the training. Specifically, the training stops if the validation score does not increase for 5 rounds. After training the ranking model, we apply the search space pruning method to find the most valuable architectural features for different tasks and hardware. There are two hyper-parameters for pruning: the sample size and the threshold. We set them to 200/1.15 and 300/1.25 for the hardwareaware architecture search and high-accuracy architecture search, respectively.

Architecture Search Setups. Table 5 and Table 6 presents the search space of high-accuracy search for the translation tasks. We refer the readers to Wang et al. (2020)'s work for more details about the

\begin{tabular}{lrcc}
\hline \multicolumn{1}{c}{ Method } & $\begin{array}{c}\text { Latency } \\
\text { (CPU) }\end{array}$ & $\begin{array}{c}\text { Latency } \\
\text { (GPU) }\end{array}$ & PPL \\
\hline Baevski and Auli (2019) & 12.49 & 0.53 & 18.70 \\
Dai et al. (2019) & 11.23 & 0.42 & 18.30 \\
Press et al. (2020) & 12.17 & 0.52 & $\mathbf{1 7 . 9 6}$ \\
\hline RankNAS (Ours) & $\mathbf{4 . 8 3}$ & $\mathbf{0 . 2 9}$ & 18.13 \\
\hline
\end{tabular}

Table 2: Performance of our discovered model and the state-of-the-art language models. The perplexities are evaluated on the WikiText-103 test data. Latency is measured in units of seconds. All models have a similar size, around 250M.

search space of hardware-aware architecture search. RankNAS is not restricted to a specific search strategy. We compare different search strategies in the experiments, including Random Search (RS) and Evolutionary Algorithm (EA). We apply uniform sampling for RS and use the same settings as Wang et al. (2020)'s work for EA. More specifically, the random search process will stop if the best-so-far architecture does not change for 3 epochs.

Evaluation Metrics. We report the results obtained by averaging 5 runs with different seeds. We calculate BLEU scores with case-sensitive tokenization using Moses, and apply the compound splitting BLEU for WMT, the same as HAT. We test the latency of models on an Intel Xeon Silver 4114 CPU and an NVIDIA GTX 1080Ti GPU. A 


\begin{tabular}{lccc|ccc}
\hline & \multicolumn{3}{c}{ ISWLT'14 De-En } & \multicolumn{3}{c}{ WMT'14 En-De } \\
\hline \multicolumn{1}{c}{ Method } & \#Params & BLEU & $\begin{array}{c}\text { Search Cost } \\
\text { (hours) }\end{array}$ & \#Params & BLEU & $\begin{array}{c}\text { Search Cost } \\
\text { (hours) }\end{array}$ \\
\hline Vaswani et al. (2017) & $35 \mathrm{M}$ & 34.4 & - & $213 \mathrm{M}$ & 28.4 & - \\
Shaw et al. (2018) & $37 \mathrm{M}$ & 35.4 & - & $213 \mathrm{M}$ & 29.2 & - \\
Wu et al. (2019b) & $43 \mathrm{M}$ & 35.2 & - & $213 \mathrm{M}$ & 29.7 & - \\
\hline Pham and Le (2021) & $37 \mathrm{M}$ & 35.8 & - & - & - & - \\
So et al. (2019) & - & - & - & $218 \mathrm{M}$ & 29.8 & $5.5 \times 10^{6}$ \\
Fan et al. (2020) & $38 \mathrm{M}$ & 36.1 & 262.7 & $213 \mathrm{M}$ & $\mathbf{3 0 . 1}$ & 1970.3 \\
Zhao et al. (2021) & - & - & - & $215 \mathrm{M}$ & 29.8 & 798.3 \\
RankNAS (Ours) & $\mathbf{3 4 M}$ & $\mathbf{3 6 . 2}$ & $\mathbf{2 . 3}$ & $\mathbf{2 0 2 M}$ & 29.9 & $\mathbf{3 6 . 9}$ \\
\hline
\end{tabular}

Table 3: Results on the IWSLT'14 De-En and WMT'14 En-De machine translation tasks. The models above are both designed by human experts, while the models below are discovered by NAS. Search costs are normalized to GPU hours on a single RTX 2080Ti GPU, according to the results on public benchmarks ${ }^{1}$.

machine translation model's latency is the time of translating a single sentence with a fixed length - 30 for WMT and 23 for IWSLT. For language modeling, the latency is the cost of decoding a single sentence without mini-batching, averaged over the whole test set. Following Wang et al. (2020)'s work, we measure each model's latency 300 times and remove the fastest and slowest $10 \%$ and then take the average of the rest $80 \%$. Note that we report the total number of trainable parameters in a model, while Wang et al. (2020) emit the parameters of the embedding layers. The search cost is the GPU hours measured on or normalized to a single RTX 2080Ti.

\subsection{Results}

Hardware-Aware Architecture Search. The hardware-aware NAS aims to maximize the accuracy under specified latency constraints on different hardware platforms. We first rank architectures by their latencies and pick those that meet the constraint to achieve this goal. Then we rank the selected architectures by their losses on the validation set and choose the best one. For machine translation tasks, we use the same search space as HAT (Wang et al., 2020), which contains around $10^{15}$ possible architectures. For the language modeling task, we use the following search space: $[10,12$, 14] for decoder layer number, [768, 1024] for embedding dimension, [3072, 4096, 5120] for hidden dimension, and $[8,12,16]$ for the head number in attention modules. We add a simple linear projection without bias if two adjacent layers have different hidden sizes.
Table 1 shows the results of RankNAS comparing to HAT (Wang et al., 2020) and Transformer (Vaswani et al., 2017) on the machine translation tasks. Our method is effective in reducing the search cost for different tasks and hardware platforms. For instance, it requires $10.53 \times$ less cost to find a comparable architecture on the WMT task. The discovered architectures also have the lowest latencies with the same or better BLEU scores on most tasks. For example, the architecture designed for the CPU is $2.68 \times$ faster than the standard Transformer.

We present the architecture search results for language modeling on the WikiText-103 test data in Table 2. All models are evaluated with a context window of 2,560 tokens, following Baevski and Auli (2019). Our method significantly accelerates the baseline on different devices. Specifically, our method speeds up the baseline by $2.59 \times$ on the CPU and $1.83 \times$ on the GPU. Our model also obtains a perplexity of 18.13 , which outperforms Transformer-XL (Dai et al., 2019) and is comparable to the state-of-the-art language model, e.g., Sandwich-Transformer (Press et al., 2020).

High-Accuracy Architecture Search. Unlike hardware-aware architecture search, the highaccuracy architecture search only optimizes accuracy and does not consider latency. In the experiments, we enlarge the HAT's search space by introducing two additional features Relative Attention Position (Shaw et al., 2018) and Layer Norm Position, as shown in Table 5 and Table 6. This expands the size of search space to $10^{23}, 8$ orders 


\begin{tabular}{cccc}
\hline $\begin{array}{c}\text { Search } \\
\text { Space }\end{array}$ & Method & Kendall's $\boldsymbol{\tau}$ & Spearman's $\boldsymbol{\rho}$ \\
\hline \multirow{2}{*}{ Small } & HAT & 0.827 & 0.913 \\
& Ours & $\mathbf{0 . 8 8 3}$ & $\mathbf{0 . 9 4 5}$ \\
\hline \multirow{2}{*}{ Large } & HAT & 0.754 & 0.842 \\
& Ours & $\mathbf{0 . 8 2 6}$ & $\mathbf{0 . 9 0 7}$ \\
\hline
\end{tabular}

Table 4: RankNAS vs. HAT in terms of Kendall and Spearman rank correlation coefficient. The results are collected using the settings described in Sec. 5.1.

of magnitude larger than HAT.

We compare RankNAS with state-of-the-art machine translation models designed by human experts and models discovered by other NAS methods. The results are presented in Table 3. RankNAS consistently outperforms other methods in both the IWSLT and WMT tasks. It demonstrates that RankNAS can also design high-accuracy architectures. Notably, the discovered architectures achieve a +1.8 BLEU improvement on the IWSLT task and $\mathrm{a}+1.5$ BLEU improvement on the WMT task than the standard Transformers baseline (Vaswani et al., 2017). We show that RankNAS surpasses the Evolved Transformer (So et al., 2019), with orders of magnitude fewer search costs. RankNAS also matches the performance of gradient-based methods, including NAO (Fan et al., 2020) and DARTSformer (Zhao et al., 2021).

\section{Analysis}

We analyze both the accuracy and efficiency of our search method and study the effect of different features on model performance.

\subsection{Architecture Ranking Accuracy}

To study the accuracy of the proposed method, we evaluate it on the IWSLT translation task. In the experiment, we randomly sample 200 different architectures from the HAT search space (small) and the enlarged search space (large) introduced in Sec. 4.2. We train these architectures from scratch and measure their BLEU scores on the test set. Table 4 presents the Kendall and Spearman rank correlation coefficient between the predicted results and the real scores. It shows that RankNAS outperforms HAT in terms of different ranking correlations. For example, RankNAS achieves a high Kendall's Tau of 0.883 and 0.826 on small and large spaces. This indicates that the predicted ranking is very close to the real results.

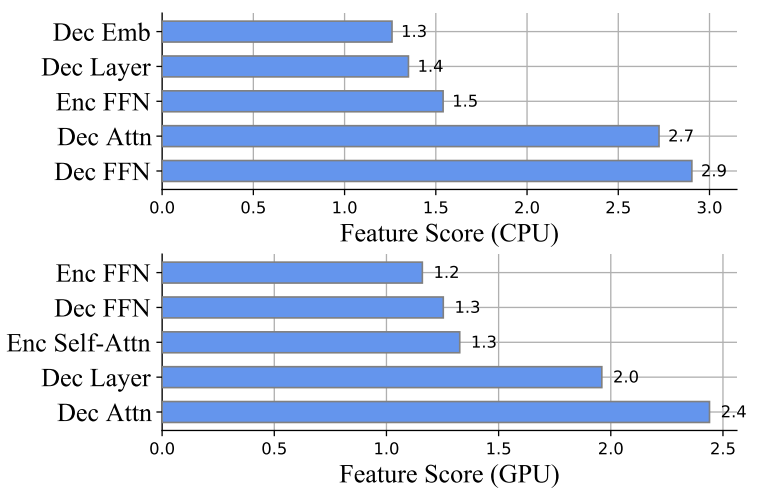

Figure 5: The selected features for different hardware platforms. A higher score means the feature is more important than others.

Importance of Ranking Accuracy. Although our ranking model is more accurate than prior methods, a question remains: how does ranking accuracy affect the search quality? We analyze the impact of different ranking models on the highaccuracy NAS task. Figure 6 compares two ranking models with different ranking correlation coefficients. The results are obtained by best-so-far models trained from scratch on the IWSLT' 14 DeEn data. Results show that inaccurate ranking leads to poor search results. It indicates that an accurate ranking model is essential for architecture search.

\subsection{Analysis of Discovered Architectures}

We present the discovered architectures in Appendix A.2 and analyze important features for different hardware on the IWSLT' 14 De-En task.

Figure 5 (top) plots the selected features for the CPU. It shows that the decoder FFN dimension is the most important feature for predicting latency, followed by the decoder's arbitrary attention and the encoder FFN dimension. We also find that the decoder embedding dimension has a similar impact on latency as the number of decoder layers.

Figure 5 (bottom) illustrates the results for the GPU. Similar to the CPU, the latency on the GPU has a high correlation to the decoder attention module. The main difference is that the latency on GPU is insensitive to FFN or embedding dimensions but more sensitive to the number of decoder layers.

The results indicate that we can design "shallow and wide" models for GPUs and "deep and thin" models for CPUs to achieve the Pareto-optimal state. Similar design insights have been verified in 


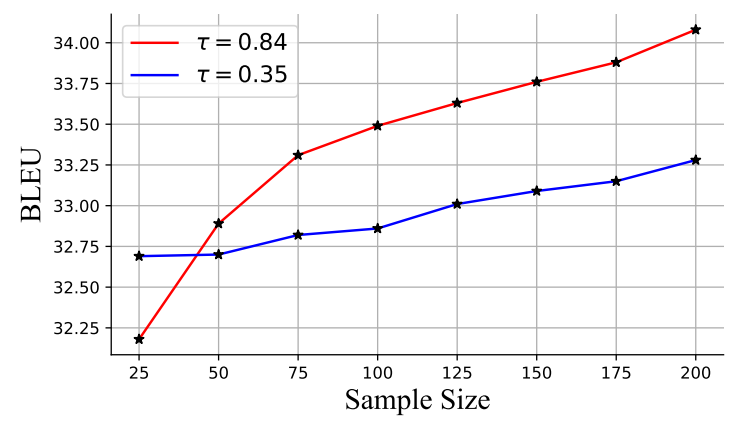

Figure 6: Search results of different ranking models. The inaccurate ranking model (in blue) leads to worse search results than the accurate ranking model (in red).

recent works, such as Wang et al. (2019), Hu et al. (2020), Li et al. (2021), and Lin et al. (2021).

\subsection{Search Efficiency}

Experiments in Sec. 4 show that our method has much lower search costs than previous works. We now analyze how does our method accelerates the architecture search.

Ranking Model Training Efficiency. The overall search cost includes the training time of the ranking model and the cost of the search process. Figure 1 compares our method and HAT on the IWSLT'14 De-En task. The two methods share the same search space and sampling strategy for search. We observe that the ranking model training takes most of the time. RankNAS speeds up the ranking model training by 10.34 times compared with HAT. Pruning the search space further reduces the $75 \%$ time of the search process. Thus the overall search cost is significantly reduced. It indicates that efficient training of the ranking model is essential to accelerate the search process.

Architecture Search Efficiency. We also analyze the efficiency of our proposed methods on the IWSLT hardware-aware task. Figure 7 shows the loss curves on the validation set of the models found by our method with different sampling strategies. We observe that RankNAS is compatible with different strategies. Also, the evolutionary algorithm outperforms random search in terms of the rate of convergence and the search result.

\section{Related Work}

Many efforts have been made to improve the NAS efficiency for different tasks (Tan et al., 2019; Wu et al., 2019a; Cai et al., 2019; Lu et al., 2019; Chen

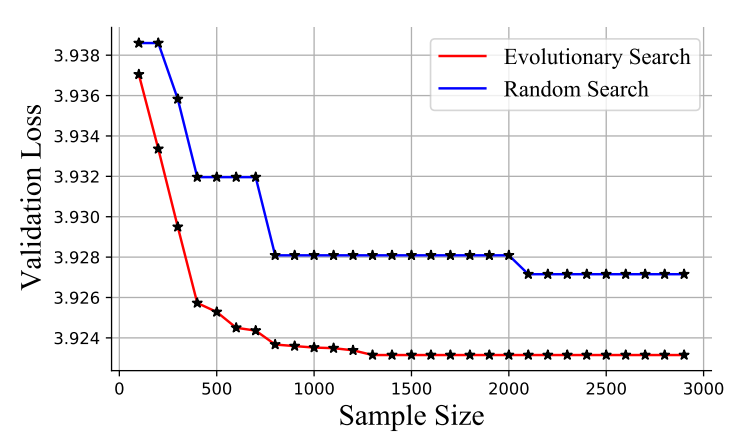

Figure 7: RankNAS combined with an evolutionary strategy achieves faster convergence and better results than other search methods.

et al., 2020). A common approach to accelerating the search process is to use a proxy, such as reduced model size, training data, or training steps. However, it is inaccurate for estimating the model's performance and diminishes the NAS quality (Baker et al., 2018; Dudziak et al., 2020). Another popular way is to share parameters among all architectures to reduce the training time (Tan et al., 2019; Cai et al., 2019). However, it is infeasible to train all architecture candidates fairly to obtain their accurate performance.

Recent works explored performance prediction based on architectural properties, i.e., the network topology and the model size (Liu et al., 2018; Long et al., 2019; Wen et al., 2020; Ning et al., 2020). For instance, Hardware-Aware Transformer (HAT) (Wang et al., 2020) encoded architectures into feature vectors and predicted the latency with a Multilayer Perceptron (MLP) for the target hardware. BRP-NAS (Dudziak et al., 2020) proposed an end-to-end performance predictor based on a Graph Convolutional Network (GCN). Although these methods greatly improve the performance estimation efficiency, they still require many samples and train numerous neural networks to converge, thereby increasing the search cost. Instead, we are motivated by the fact that NAS is expected to distinguish different candidate architectures. Thus, NAS can be solved by learning pairwise ranking rather than obtaining the accurate performance of architectures.

\section{Conclusion}

We have presented RankNAS, a simple yet efficient NAS algorithm for both hardware-aware and highaccuracy architecture search. We have shown that pairwise ranking can significantly improve search 
efficiency. We also have proposed a search space pruning method to help the ranking model be more efficient during the search. Our approach outperforms prior methods in both efficiency and accuracy. RankNAS requires $80 \%$ less time in ranking model training on the hardware-aware search task and accelerates the overall search process by 11.53 times. Also, the architectures discovered by our method outperform state-of-the-art Transformer models in terms of efficiency and accuracy.

\section{Acknowledgements}

This work was supported in part by the National Science Foundation of China (Nos. 61876035 and 61732005), the National Key R\&D Program of China (No.2019QY1801), and the Ministry of Science and Technology of the PRC (Nos. 2019YFF0303002 and 2020AAA0107900). The authors would like to thank the anonymous reviewers for their comments and suggestions.

\section{References}

Alexei Baevski and Michael Auli. 2019. Adaptive input representations for neural language modeling. In 7 th International Conference on Learning Representations, ICLR 2019, New Orleans, LA, USA, May 6-9, 2019. OpenReview.net.

Bowen Baker, Otkrist Gupta, Ramesh Raskar, and Nikhil Naik. 2018. Accelerating neural architecture search using performance prediction. In 6 th International Conference on Learning Representations, ICLR 2018, Vancouver, BC, Canada, April 30 - May 3, 2018, Workshop Track Proceedings. OpenReview.net.

Leo Breiman. 2001. Random forests. Mach. Learn., 45(1):5-32.

Christopher J. C. Burges, Tal Shaked, Erin Renshaw, Ari Lazier, Matt Deeds, Nicole Hamilton, and Gregory N. Hullender. 2005. Learning to rank using gradient descent. In Machine Learning, Proceedings of the Twenty-Second International Conference (ICML 2005), Bonn, Germany, August 7-11, 2005, volume 119 of ACM International Conference Proceeding Series, pages 89-96. ACM.

Han Cai, Tianyao Chen, Weinan Zhang, Yong Yu, and Jun Wang. 2018. Efficient architecture search by network transformation. In Proceedings of the ThirtySecond AAAI Conference on Artificial Intelligence, (AAAI-18), the 30th innovative Applications of Artificial Intelligence (IAAI-18), and the 8th AAAI Symposium on Educational Advances in Artificial Intelligence (EAAI-18), New Orleans, Louisiana, USA, February 2-7, 2018, pages 2787-2794. AAAI Press.
Han Cai, Ligeng Zhu, and Song Han. 2019. Proxylessnas: Direct neural architecture search on target task and hardware. In 7th International Conference on Learning Representations, ICLR 2019, New Orleans, LA, USA, May 6-9, 2019. OpenReview.net.

Daoyuan Chen, Yaliang Li, Minghui Qiu, Zhen Wang, Bofang Li, Bolin Ding, Hongbo Deng, Jun Huang, Wei Lin, and Jingren Zhou. 2020. Adabert: Taskadaptive BERT compression with differentiable neural architecture search. In Proceedings of the Twenty-Ninth International Joint Conference on Artificial Intelligence, IJCAI 2020, pages 2463-2469. ijcai.org.

Zihang Dai, Zhilin Yang, Yiming Yang, Jaime Carbonell, Quoc Le, and Ruslan Salakhutdinov. 2019. Transformer-XL: Attentive language models beyond a fixed-length context. In Proceedings of the 57th Annual Meeting of the Association for Computational Linguistics, pages 2978-2988, Florence, Italy. Association for Computational Linguistics.

Lukasz Dudziak, Thomas C. P. Chau, Mohamed S. Abdelfattah, Royson Lee, Hyeji Kim, and Nicholas D. Lane. 2020. BRP-NAS: prediction-based NAS using gens. In Advances in Neural Information Processing Systems 33: Annual Conference on Neural Information Processing Systems 2020, NeurIPS 2020, December 6-12, 2020, virtual.

Yang Fan, Fei Tian, Yingce Xia, Tao Qin, Xiang-Yang $\mathrm{Li}$, and Tie-Yan Liu. 2020. Searching better architectures for neural machine translation. IEEE ACM Trans. Audio Speech Lang. Process., 28:1574-1585.

Aaron Fisher, Cynthia Rudin, and Francesca Dominici. 2019. All models are wrong, but many are useful: Learning a variable's importance by studying an entire class of prediction models simultaneously. $J$. Mach. Learn. Res., 20:177:1-177:81.

Chi Hu, Bei Li, Yinqiao Li, Ye Lin, Yanyang Li, Chenglong Wang, Tong Xiao, and Jingbo Zhu. 2020. The NiuTrans system for WNGT 2020 efficiency task. In Proceedings of the Fourth Workshop on Neural Generation and Translation, pages 204-210, Online. Association for Computational Linguistics.

Yufan Jiang, Chi Hu, Tong Xiao, Chunliang Zhang, and Jingbo Zhu. 2019. Improved differentiable architecture search for language modeling and named entity recognition. In Proceedings of the 2019 Conference on Empirical Methods in Natural Language Processing and the 9th International Joint Conference on Natural Language Processing (EMNLPIJCNLP), pages 3585-3590, Hong Kong, China. Association for Computational Linguistics.

Guolin Ke, Qi Meng, Thomas Finley, Taifeng Wang, Wei Chen, Weidong Ma, Qiwei Ye, and Tie-Yan Liu. 2017. Lightgbm: A highly efficient gradient boosting decision tree. In Advances in Neural Information Processing Systems 30: Annual Conference on 
Neural Information Processing Systems 2017, December 4-9, 2017, Long Beach, CA, USA, pages 3146-3154.

Yanyang Li, Ye Lin, Tong Xiao, and Jingbo Zhu. 2021 An efficient transformer decoder with compressed sub-layers. In Thirty-Fifth AAAI Conference on Artificial Intelligence, AAAI 2021, Thirty-Third Conference on Innovative Applications of Artificial Intelligence, IAAI 2021, The Eleventh Symposium on Educational Advances in Artificial Intelligence, EAAI 2021, Virtual Event, February 2-9, 2021, pages 13315-13323. AAAI Press.

Yinqiao Li, Chi Hu, Yuhao Zhang, Nuo Xu, Yufan Jiang, Tong Xiao, Jingbo Zhu, Tongran Liu, and Changliang Li. 2020. Learning architectures from an extended search space for language modeling. In Proceedings of the 58th Annual Meeting of the Association for Computational Linguistics, pages 66296639, Online. Association for Computational Linguistics.

Ye Lin, Yanyang Li, Ziyang Wang, Bei Li, Quan Du, Tong Xiao, and Jingbo Zhu. 2021. Weight distillation: Transferring the knowledge in neural network parameters. In Proceedings of the 59th Annual Meeting of the Association for Computational Linguistics and the 11th International Joint Conference on Natural Language Processing (Volume 1: Long Papers), pages 2076-2088, Online. Association for Computational Linguistics.

Chenxi Liu, Barret Zoph, Maxim Neumann, Jonathon Shlens, Wei Hua, Li-Jia Li, Li Fei-Fei, Alan L. Yuille, Jonathan Huang, and Kevin Murphy. 2018. Progressive neural architecture search. In Computer Vision - ECCV 2018 - 15th European Conference, Munich, Germany, September 8-14, 2018, Proceedings, Part I, volume 11205 of Lecture Notes in Computer Science, pages 19-35. Springer.

Hanxiao Liu, Karen Simonyan, and Yiming Yang 2019. DARTS: differentiable architecture search. In 7th International Conference on Learning Representations, ICLR 2019, New Orleans, LA, USA, May 6-9, 2019. OpenReview.net.

D. Long, S. Zhang, and Y. Zhang. 2019. Performance prediction based on neural architecture features. In 2019 2nd China Symposium on Cognitive Computing and Hybrid Intelligence (CCHI), pages 77-80.

Qing Lu, Weiwen Jiang, Xiaowei Xu, Yiyu Shi, and Jingtong $\mathrm{Hu}$. 2019. On neural architecture search for resource-constrained hardware platforms. CoRR, $\mathrm{abs} / 1911.00105$.

Stephen Merity, Caiming Xiong, James Bradbury, and Richard Socher. 2017. Pointer sentinel mixture models. In 5th International Conference on Learning Representations, ICLR 2017, Toulon, France, April 24-26, 2017, Conference Track Proceedings. OpenReview.net.
Xuefei Ning, Yin Zheng, Tianchen Zhao, Yu Wang, and Huazhong Yang. 2020. A generic graph-based neural architecture encoding scheme for predictorbased NAS. In Computer Vision - ECCV 2020 16th European Conference, Glasgow, UK, August 23-28, 2020, Proceedings, Part XIII, volume 12358 of Lecture Notes in Computer Science, pages 189204. Springer.

Hieu Pham, Melody Y. Guan, Barret Zoph, Quoc V. Le, and Jeff Dean. 2018. Efficient neural architecture search via parameter sharing. In Proceedings of the 35th International Conference on Machine Learning, ICML 2018, Stockholmsmässan, Stockholm, Sweden, July 10-15, 2018, volume 80 of Proceedings of Machine Learning Research, pages 4092-4101. PMLR.

Hieu Pham and Quoc V. Le. 2021. Autodropout: Learning dropout patterns to regularize deep networks. In Thirty-Fifth AAAI Conference on Artificial Intelligence, AAAI 2021, Thirty-Third Conference on Innovative Applications of Artificial Intelligence, IAAI 2021, The Eleventh Symposium on Educational Advances in Artificial Intelligence, EAAI 2021, Virtual Event, February 2-9, 2021, pages 9351-9359. AAAI Press.

Ofir Press, Noah A. Smith, and Omer Levy. 2020. Improving transformer models by reordering their sublayers. In Proceedings of the 58th Annual Meeting of the Association for Computational Linguistics, pages 2996-3005, Online. Association for Computational Linguistics.

Esteban Real, Alok Aggarwal, Yanping Huang, and Quoc V. Le. 2019. Regularized evolution for image classifier architecture search. In The ThirtyThird AAAI Conference on Artificial Intelligence, AAAI 2019, The Thirty-First Innovative Applications of Artificial Intelligence Conference, IAAI 2019, The Ninth AAAI Symposium on Educational Advances in Artificial Intelligence, EAAI 2019, Honolulu, Hawaii, USA, January 27 - February 1, 2019, pages 4780-4789. AAAI Press.

Peter Shaw, Jakob Uszkoreit, and Ashish Vaswani. 2018. Self-attention with relative position representations. In Proceedings of the 2018 Conference of the North American Chapter of the Association for Computational Linguistics: Human Language Technologies, Volume 2 (Short Papers), pages 464-468, New Orleans, Louisiana. Association for Computational Linguistics.

David R. So, Quoc V. Le, and Chen Liang. 2019. The evolved transformer. In Proceedings of the 36th International Conference on Machine Learning, ICML 2019, 9-15 June 2019, Long Beach, California, USA, volume 97 of Proceedings of Machine Learning Research, pages 5877-5886. PMLR.

Mingxing Tan, Bo Chen, Ruoming Pang, Vijay Vasudevan, Mark Sandler, Andrew Howard, and Quoc V. Le. 2019. Mnasnet: Platform-aware neural architecture search for mobile. In IEEE Conference on Computer Vision and Pattern Recognition, CVPR 2019, 
Long Beach, CA, USA, June 16-20, 2019, pages 2820-2828. Computer Vision Foundation / IEEE.

Ashish Vaswani, Noam Shazeer, Niki Parmar, Jakob Uszkoreit, Llion Jones, Aidan N. Gomez, Lukasz Kaiser, and Illia Polosukhin. 2017. Attention is all you need. In Advances in Neural Information Processing Systems 30: Annual Conference on Neural Information Processing Systems 2017, December 49, 2017, Long Beach, CA, USA, pages 5998-6008.

Hanrui Wang, Zhanghao Wu, Zhijian Liu, Han Cai, Ligeng Zhu, Chuang Gan, and Song Han. 2020. HAT: Hardware-aware transformers for efficient natural language processing. In Proceedings of the 58th Annual Meeting of the Association for Computational Linguistics, pages 7675-7688, Online. Association for Computational Linguistics.

Qiang Wang, Bei Li, Tong Xiao, Jingbo Zhu, Changliang Li, Derek F. Wong, and Lidia S. Chao. 2019. Learning deep transformer models for machine translation. In Proceedings of the 57th Annual Meeting of the Association for Computational Linguistics, pages 1810-1822, Florence, Italy. Association for Computational Linguistics.

Fabian L. Wauthier, Michael I. Jordan, and Nebojsa Jojic. 2013. Efficient ranking from pairwise comparisons. In Proceedings of the 30th International Conference on Machine Learning, ICML 2013, Atlanta, GA, USA, 16-21 June 2013, volume 28 of JMLR Workshop and Conference Proceedings, pages 109117. JMLR.org.

Chen Wei, Chuang Niu, Yiping Tang, and Jimin Liang. 2020. NPENAS: neural predictor guided evolution for neural architecture search. CoRR, abs/2003.12857.

Wei Wen, Hanxiao Liu, Yiran Chen, Hai Helen Li, Gabriel Bender, and Pieter-Jan Kindermans. 2020. Neural predictor for neural architecture search. In Computer Vision - ECCV 2020 - 16th European Conference, Glasgow, UK, August 23-28, 2020, Proceedings, Part XXIX, volume 12374 of Lecture Notes in Computer Science, pages 660-676. Springer.

Bichen Wu, Xiaoliang Dai, Peizhao Zhang, Yanghan Wang, Fei Sun, Yiming Wu, Yuandong Tian, Peter Vajda, Yangqing Jia, and Kurt Keutzer. 2019a. Fbnet: Hardware-aware efficient convnet design via differentiable neural architecture search. In IEEE Conference on Computer Vision and Pattern Recognition, CVPR 2019, Long Beach, CA, USA, June 16-20, 2019, pages 10734-10742. Computer Vision Foundation / IEEE.

Felix Wu, Angela Fan, Alexei Baevski, Yann N. Dauphin, and Michael Auli. 2019b. Pay less attention with lightweight and dynamic convolutions. In 7th International Conference on Learning Representations, ICLR 2019, New Orleans, LA, USA, May 6-9, 2019. OpenReview.net.
Ruibin Xiong, Yunchang Yang, Di He, Kai Zheng, Shuxin Zheng, Chen Xing, Huishuai Zhang, Yanyan Lan, Liwei Wang, and Tie-Yan Liu. 2020. On layer normalization in the transformer architecture. In Proceedings of the 37th International Conference on Machine Learning, ICML 2020, 13-18 July 2020, Virtual Event, volume 119 of Proceedings of Machine Learning Research, pages 10524-10533. PMLR.

Yuekai Zhao, Li Dong, Yelong Shen, Zhihua Zhang, Furu Wei, and Weizhu Chen. 2021 Memory-efficient differentiable transformer architecture search. In Findings of the Association for Computational Linguistics: ACL/IJCNLP 2021, Online Event, August 1-6, 2021, volume ACL/IJCNLP 2021 of Findings of ACL, pages 4254-4264. Association for Computational Linguistics.

Zhao Zhong, Junjie Yan, Wei Wu, Jing Shao, and Cheng-Lin Liu. 2018. Practical block-wise neural network architecture generation. In 2018 IEEE Conference on Computer Vision and Pattern Recognition, CVPR 2018, Salt Lake City, UT, USA, June 1822, 2018, pages 2423-2432. IEEE Computer Society.

Barret Zoph, Vijay Vasudevan, Jonathon Shlens, and Quoc V. Le. 2018. Learning transferable architectures for scalable image recognition. In 2018 IEEE Conference on Computer Vision and Pattern Recognition, CVPR 2018, Salt Lake City, UT, USA, June 18-22, 2018, pages 8697-8710. IEEE Computer Society. 


\section{A Appendix}

\section{A.1 High-Accuracy Architecture Search Space}

Other design choices are adopted from HAT's search space (Wang et al., 2020) with slight modifications. Inspired by Shaw et al. (2018), we search for the maximum relative position ( $R P R$ Len) in the self-attention modules in each layer. As suggested by Wang et al. (2019) and Xiong et al. (2020), proper locations of layer normalization lead to better performance. Therefore, we let NAS decide whether to put the layer normalization inside (PreLN) or between (Post-LN) the residual blocks.

\begin{tabular}{c|c}
\hline Features & Search Space \\
\hline Enc Layer Num & {$[6]$} \\
Enc Emb Dim & {$[512,640,768]$} \\
Enc FFN Dim & {$[768,1024,1536,2048]$} \\
Enc Head Num & {$[2,4,8]$} \\
Enc RPR Len & {$[8,12,16]$} \\
Enc Norm Type & {$[$ Pre-LN, Post-LN] } \\
Dec Layer Num & {$[1,2,3,4,5,6]$} \\
Dec Emb Dim & {$[512,640,768]$} \\
Dec FFN Dim & {$[768,1024,1536,2048]$} \\
Dec Head Num & {$[2,4,8]$} \\
Dec RPR Len & {$[8,12,16]$} \\
Dec Norm Type & {$[$ Pre-LN, Post-LN] } \\
Enc-Dec Attn & {$[1,2,3]$} \\
\hline
\end{tabular}

Table 5: The search space for high-accuracy search on the IWSLT'14 De-En translation task.

\begin{tabular}{c|c}
\hline Features & Search Space \\
\hline Enc Layer Num & {$[6]$} \\
Enc Emb Dim & {$[640,768,1024]$} \\
Enc FFN Dim & {$[2048,3072,4096,5120]$} \\
Enc Head Num & {$[4,8,16]$} \\
Enc RPR Len & {$[8,12,16]$} \\
Enc Norm Type & {$[$ Pre-LN, Post-LN $]$} \\
Dec Layer Num & {$[1,2,3,4,5,6]$} \\
Dec Emb Dim & {$[640,768,1024]$} \\
Dec FFN Dim & {$[2048,3072,4096,5120]$} \\
Dec Head Num & {$[4,8,16]$} \\
Dec RPR Len & {$[8,12,16]$} \\
Dec Norm Type & {$[$ Pre-LN, Post-LN $]$} \\
Enc-Dec Attn & {$[1,2,3]$} \\
\hline
\end{tabular}

Table 6: The search space for high-accuracy search on the WMT'14 En-De translation task.

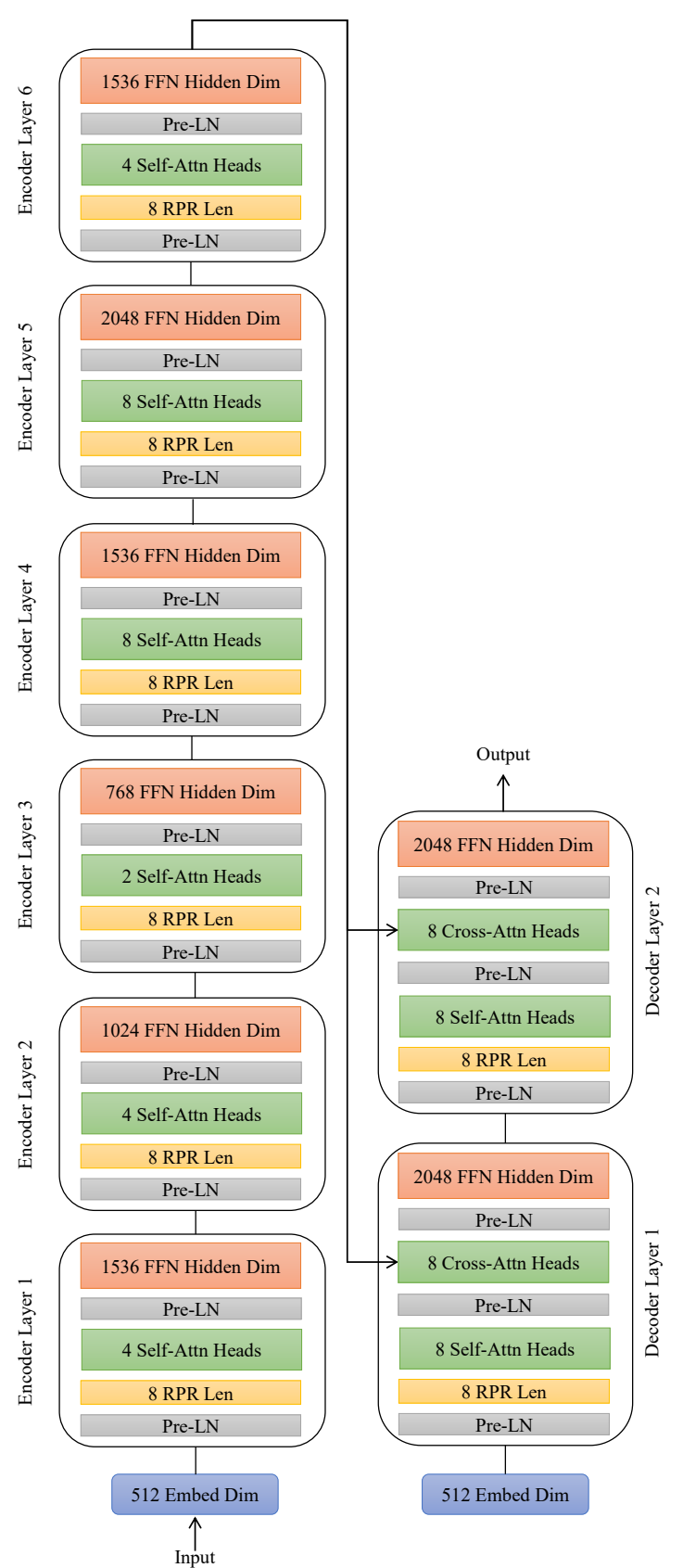

Figure 8: Visualization of a discovered architecture on the IWSLT'14 De-En translation task.

\section{A.2 Visualization of Good Architectures}

Figure 8 illustrates one of the discovered Transformer architecture. The presented architecture achieves 36.2 BLEU on the IWSLT'14 De-En translation task and has a latency of $77 \mathrm{~ms}$ on the GTX 1080Ti GPU, outperforming the vanilla Transformer by +1.8 BLEU and 2.6 times speed. 